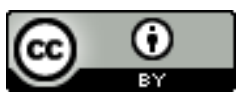

Esta obra está sob o direito de Licença Creative Commons Atribuição 4.0 Internacional.

\title{
A ATUAÇÃO DO PSICÓLOGO NO CONTEXTO HOSPITALAR
}

\author{
Maria Verônica Tenório de Araújo ${ }^{1}$ \\ Eduardo Cabral da Silvar ${ }^{2}$ \\ Maria Edleuza da Paz \\ Maria Isabel da Silva Martins ${ }^{4}$
}

\section{RESUMO}

Este artigo aborda um estudo desenvolvido a acerca da atuação do psicólogo no contexto hospitalar, pois o trabalho desenvolvido pelos profissionais da psicologia nas instituições hospitalares, seja no âmbito público ou privado, tem aumentado de forma significativa a cada dia. A pesquisa foi desenvolvida na perspectiva de responder ao seguinte problema: Qual é o papel do psicólogo no contexto hospitalar? Por meio da realização de uma revisão sistemática integrativa, desenvolvida a partir da exploração de publicação científicas entre os anos de 2010 à 2020. O que permitiu compreender que que o suporte psicológico ao paciente, e aos familiares é essencial, o que permite compreender a relevância do psicólogo hospitalar para a promoção do auxílio ao tratamento e a compreensão da internação e do adoecimento, assim como também em situações de óbito.

Palavras-chave: Psicologia. Saúde. Hospitalar.

\footnotetext{
${ }^{1}$ veronicatenorioa@gmail.com

2 edcs.cabral@gmail.com

3 mariaedleuza.leu@gmail.com

4 isabell.maria2011@gmail.com
} 


\section{INTRODUÇÃO}

O trabalho desenvolvido pelos profissionais da psicologia nas instituições hospitalares, seja no âmbito público ou privado, tem aumentado de forma significativa a cada dia. No entanto, ainda está firmando sua identidade nesse contexto, pela multiplicidade de tarefas que ocupa e seu caráter multiprofissional.

Vale salientar que a psicologia contribui com o serviço realizado por toda equipe multiprofissional que atua no contexto hospitalar, frente aos diversos fatores precipitantes e predisponentes do processo de adoecimento. De acordo com Assis (2019, s/p.):

Nessa área de atuação percebe-se que as funções desempenhadas pelos psicólogos são muito mais abrangentes do que nos modelos tradicionais de atendimento.

Conforme Mota, Martins e Veras (2006) a psicologia da área da saúde exerce relevante contribuição não apenas com uma visão da recuperação, mas também, com a inserção da promoção da saúde, que visa a prevenção de doenças e a manutenção da saúde nos aspectos social, físico, espiritual e mental.

Fatores como o avanço das diversas áreas da saúde, nos últimos anos que iniciaram o século XXI, observados a partir da ampliação na sofisticação dos recursos e técnicas de tratamento e diagnósticos, a psicologia tem oferecidos aos diversos profissionais que constituem a equipe multidisciplinar hospitalar, um apoio indispensável, tendo em vista, que a sua atuação no contexto hospitalar centra-se na minimização do sofrido provocado ao paciente e aos seus familiares pela enfermidade causadora da hospitalização (ANGERAMI, 2011).

A presença efetiva do psicólogo no contexto hospitalar se deu em 1954 no Instituto de Traumatologia (IOT) da Universidade de São Paulo (USP) por Mathilde Neder. Na perspectiva de auxiliar na preservação das pessoas no contexto hospitalar (ANGERAMI, 2011).

\begin{abstract}
A Psicologia da Saúde é a ciência que busca responder questões relativas à forma como o bem-estar das pessoas pode ser afetado pelo que se pensa, sente e faz (STRAUB, 2005, apud MOSIMANN; LUSTOSA, 2011, p. $211 / 212$ ).
\end{abstract}

Desse modo, é importante reconhecer que a Psicologia da Saúde objetiva a compreensão dos fatores sociais, biológicos e comportamentais que exercem influência no processo de saúde e doença (CASTRO; BORNHOLDT, 2004).

Desse modo, a função do psicólogo da saúde pode-se realizar em diversos 
contextos nesse âmbito, coo em instituições não governamentais, espaços domiciliares, instituições de saúde e hospitais.

Assim, é importante que se destaque as influências positivas que o psicólogo escolar exerce no contexto hospitalar, contribuindo para a o processo de recuperação da saúde e enfrentamento da enfermidade.

De acordo com o Conselho Federal de Psicologia (2007, s/p.):

O psicólogo especialista em Psicologia Hospitalar tem seu exercício profissional nos níveis secundários e terciários na atenção à saúde, instituições de ensino superior e/ou centros de estudo e de pesquisa, colaborando com a formação de outros profissionais de saúde. Faz

\section{MÉTODOLOGIA}

A metodologia adotada no presente trabalho foi a revisão sistemática. Desenvolvida a partir da exploração de publicações científicas abordando a temática. Cujo método tem por sua razão realizar resgate de estudos já publicados, ampliando assim a confiabilidade e profundidade das revisões, determinando o conhecimento atual sobre uma determinada temática especificamente, procedendo com as seguintes etapas: $1^{a}$ ) elaboração de título, pergunta norteadora, e descritores, $2^{\mathrm{a}}$ ) definição das bases de dados para busca e cruzamento entre descritores nas bases atendimentos a pacientes e familiares, suporte emocional em situações específicas a membros da equipe multiprofissional e eventualmente administrativa, além de colaborar com alunos e pesquisadores, quando estes estejam atuando em pesquisa e assistência.

Assim, o psicólogo hospitalar precisa estar sempre atento as suas funções nesse contexto, considerando os diferentes fatores que se associam à sua intervenção.

Contudo, este artigo tem como objetivo compreender o papel do psicólogo no contexto hospitalar. $\mathrm{Na}$ perspectiva de responder a seguinte questão norteadora: Qual é o papel do psicólogo no contexto hospitalar? A partir da realização desse estudo.

definidas, $3^{\text {a }}$ ) seleção dos critérios de inclusão estabelecidos da amostra, $4^{\mathrm{a}}$ ) leitura de títulos relacionados ao tema proposto, $5^{a}$ ) seleção de artigos para leitura de textos completos após análise dos resumos, $6^{\text {a }}$ ) interpretação dos resultados, reportando de forma clara as evidências encontradas.

A coleta dessas produções efetuouse por meio da Biblioteca Virtual em Saúde (BVS), sendo manuseadas nas seguintes bases de dados: Literatura LatinoAmericana e do Caribe Ciências da Saúde (LILACS), as bibliotecas virtuais Scientific Electronic Library Online (SCIELO). 
O Quadro abaixo apresenta os descritores utilizados e na busca de artigos, apontando a quantidade de publicações encontradas e as que foram utilizadas a partir dos critérios de inclusão e exclusão.

Quadro 1- Resultados de busca por descritores.

\begin{tabular}{|c|c|c|c|c|c|}
\hline ETAPA & $\begin{array}{l}\text { TÓPICOS DE } \\
\text { CADA } \\
\text { ETAPA }\end{array}$ & \multicolumn{4}{|c|}{ DETALHAMENTO DE CADA TÓPICO } \\
\hline \multirow[t]{14}{*}{$1^{\mathrm{a}}$} & Tema & \multicolumn{4}{|c|}{ A ATUAÇÃO DO PSICÓLOGO NO CONTEXTO HOSPITALAR } \\
\hline & $\begin{array}{c}\text { Pergunta } \\
\text { norteadora }\end{array}$ & \multicolumn{4}{|c|}{ Qual é o papel do psicólogo no contexto hospitalar? } \\
\hline & Objetivo geral & & & & \\
\hline & $\begin{array}{l}\text { Estratégias de } \\
\text { busca }\end{array}$ & \multicolumn{4}{|c|}{$\begin{array}{l}\text { 1. Cruzamento de descritores por meio do operador booleano AND; } \\
\text { 2. Uso de aspas nos politermos (descritor com mais de um termo) } \\
\text { para que a varredura de artigos científicos contemplasse o termo } \\
\text { exato; }\end{array}$} \\
\hline & \multirow{3}{*}{$\begin{array}{l}\text { Bancos de } \\
\text { terminologias }\end{array}$} & Banco & \multicolumn{3}{|c|}{ Link } \\
\hline & & DeSC & \multirow{2}{*}{\multicolumn{3}{|c|}{$\begin{array}{l}\text { http://decs.bvs.br/ } \\
\text { https://www.ncbi.nlm.nih.gov/mesh }\end{array}$}} \\
\hline & & $\mathrm{MeSH}$ & & & \\
\hline & \multirow{4}{*}{$\begin{array}{l}\text { Descritores } \\
\text { livres e } \\
\text { estruturados }\end{array}$} & Descritor & $\mathrm{DeC}$ & (Registro) & MeSH (Identificador Único) \\
\hline & & Psiclogia & & 009769 & D011584 \\
\hline & & Saúde & & 006297 & D006262 \\
\hline & & Hospitalar & & CS050247 & D006761 \\
\hline & String de busca & \multicolumn{4}{|c|}{ "Psicologia Hospitalar" And Saúde; } \\
\hline & \multirow{2}{*}{$\begin{array}{l}\text { Bibliotecas } \\
\text { Virtuais }\end{array}$} & \multicolumn{2}{|c|}{ Biblioteca } & \multicolumn{2}{|c|}{ Link } \\
\hline & & \multicolumn{2}{|l|}{ BVS } & \multicolumn{2}{|c|}{$\underline{\text { https://www.periodicos.capes.gov.br/ }}$} \\
\hline \multirow[t]{3}{*}{$2^{\mathrm{a}}$} & $\begin{array}{l}\text { Período de } \\
\text { coleta dos } \\
\text { dados }\end{array}$ & \multicolumn{4}{|c|}{03 de março a 10 de maio de 2020.} \\
\hline & $\begin{array}{l}\text { Critérios de } \\
\text { inclusão }\end{array}$ & \multicolumn{4}{|c|}{$\begin{array}{l}\text { 3. Artigos científicos open source } \\
\text { 4. Últimos cinco anos de publicados }\end{array}$} \\
\hline & $\begin{array}{l}\text { Critérios de } \\
\text { exclusão }\end{array}$ & \multicolumn{4}{|c|}{ 3. Artigos não relacionados a temática } \\
\hline $3^{\mathrm{a}}$ & \multicolumn{5}{|c|}{$\begin{array}{lr}\text { Número de } & \text { trabalhos } \\
\text { selecionados } & \text { para } \\
\end{array}$} \\
\hline
\end{tabular}




\begin{tabular}{|c|c|c|}
\hline & $\begin{array}{lr}\text { revisão } & \text { sistemática } \\
\text { integrativa a } & \text { partir da } \\
\text { leitura dos } & \text { agentes } \\
\text { indexadores } & \text { das } \\
\text { publicações } & \text { (tema, } \\
\text { descrição, ementa). }\end{array}$ & \\
\hline $4^{a}$ & \begin{tabular}{lr}
\multicolumn{2}{c}{ Categorias obtidas com } \\
a análise dos \\
documentos \\
investigados \\
gratuitos e de line \\
acesso
\end{tabular} & 2 \\
\hline $5^{a}$ & $\begin{array}{lr}\text { Análise, interpretação e } \\
\text { discussão } \\
\text { resultados }\end{array}$ & Ver em "Resultados e Discussão" \\
\hline $6^{a}$ & $\begin{array}{l}\text { Apresentação da } \\
\text { revisão em formato de } \\
\text { artigo, o qual contemple } \\
\text { propostas para estudos } \\
\text { futuros }\end{array}$ & Este Artigo completo \\
\hline
\end{tabular}

Fonte: autoria própria.

Os critérios de inclusão foram artigos nacionais publicados entre os anos de 2010 à 2020 contemplando discussões ligadas diretamente a temática abordada, publicado

\section{RESULTADOS E DISCUSSÕES}

No âmbito hospitalar pode-se identificar que o psicólogo faz intervenção como mediador psicológico, na perspectiva de contribuir com a relação entre equipe/paciente e equipe/família (ASSIS, 2019).

Pois, como ficou evidente a equipe e a família realizam papéis indispensáveis em todo o processo de relação entre o paciente e a sua doença, tendo em vista, que o hospitalizado compartilhará seus medos, dependências, limitações, impotências, em sites fidedignos correspondentes ao tema, que correspondiam a questão norteadora da pesquisa. A partir dos descritos pertinentes a temática.

dificuldades de adaptação ao contexto hospitalar, negação da realidade, sentimento de culpa , etc.. (PIMENTEL; LIMA; FONSECA, 2009).

De acordo com o que afirma Batista (2010), o atendimento psicológico no contexto hospitalar tem como objetivo realizar a promoção do bem-estar biopsicossocial do paciente e seus familiares, o que atualmente sob a consideração de outros aspectos foi expandido para

biopsicossocioespirituambiental, das pessoas hospitalizadas e seus familiares, o 
que exige um trabalho integrado com os demais profissionais que constituem a equipe multiprofissional hospitalar sob o enfoque da interdisciplinaridade (ANGERAMI, 2011).

Referindo-se a atuação do psicólogo no contexto hospitalar junto as crianças. Altamira (2010), aponta como objetivo a minimização do sofrimento das crianças no âmbito hospitalar, na perspectiva de diminuir a hostilidade do ambiente. $\mathrm{O}$ que independe da enfermidade enfrentada por ela.

A psicologia hospitalar é tão importante que para que atue na área o profissional de psicologia deve adquirir conhecimentos específicos, por meio de especialização, por meio de estudos mais aprofundados relacionados a prática profissional no contexto hospitalar (.

Tal concepção se dá a partir do envolvimento da compreensão dos métodos e teorias concernentes a psicologia da saúde que são aplicadas ao ambiente hospitalar.

O psicólogo hospitalar capacitado tende desenvolver seu trabalho sob a utilização de critérios que contemplem o controle da dor, a saúde física, a reabilitação de pacientes que tenham doenças crônicas, o estresse, e também no desenvolvimento de intervenções em diversos problemas, inclusive em cirurgias invasivas (OGDEN,
2007 apud ALMEIDA; MALAGRIS, 2011).

Mesmo sob o reconhecimento da importância da atuação do psicólogo hospitalar, o processo de formação desses profissionais ainda tem sido voltado para a área clínica focada no sujeito, com objetivos analíticos, psicodiagnósticos e psicoterapêuticos.

A sua inserção, sem que haja a devida preparação para que atue no ambiente hospitalar, pode incidir na aplicabilidade do falso saber, comprometendo assim a eficiência do serviço psicológico disponibilizado ao paciente. Além de dificultar a viabilização do dinamismo na relação entre a equipe multiprofissional e o doente.

Assim, pode-se identificar ainda que a inserção da Psicologia nos hospitais gerais teve seu início a partir do ano de 1954 e 1957, a partir da inclusão do serviço de atendimento psicológico no Hospital das Clínicas da Faculdade de Medicina de São Paulo (SEBASTIANI, 2000).

Nesse período, o Serviço de Psicologia ofertado no Instituto de Traumatologia do Hospital das Clínicas, estava voltado para a preparação psicológica das crianças que iriam realizar cirurgia do aparelho locomotor. Constituindo-se como uma iniciativa restrita no cenário brasileiro. 
Com isso, observou-se que considerando a importância da psicologia hospitalar e da formação desses profissionais, essa área da psicologia vem se expandindo ao longo dos anos, valendo respaldar que as primeiras disciplinas inerentes a saúde foram ministradas inicialmente nos cursos de psicologia da Universidade de São Paulo (TOREZAN et al., 2013).

Desde então, a psicologia hospitalar vem se expandindo e construindo sua identidade profissional, tendo o seu trabalho delineado a partir da percepção dos enfermeiros e dos médicos de que algumas situações fogem de seus conhecimentos (TOREZAN et al., 2013).

Adjacente ao enfoque que tange sobre a humanização da assistência ao paciente, a partir da instituição do trabalho em equipe multidisciplinar, que consiste como uma das bases que permite a integração das atividades desenvolvidas pelo psicólogo nesse contexto, sob a consideração do paciente como indivíduo biopsicossocial (TAVARES et al., 2012).

De acordo com Sebastiani e Maia (2010), a atuação do psicólogo no contexto hospitalar se dá na minimização da ansiedade e da angústia do paciente hospitalizado, "favorecendo a expressão dos sentimentos e auxiliando na compreensão da situação vivenciada", oportunizando o reforço da confiança entre a equipe de saúde e o paciente. Fazendo-se necessário a sua intervenção no processo de reorganização da consciência do paciente, indispensáveis para que seja reestruturado o autoconceito.

Referindo-se de forma extremamente direta a atuação do psicólogo, também é importante considerar a colocação de Romano (1999) apud Baptista (2010, p.180) ao afirmar que “o psicólogo no ambiente hospitalar deve ser um observador qualificado, além de ser intérprete flexível dos anseios do paciente da sua família e das normas da instituição, sendo encarado como um agente de transformação no processo de reabilitação".

O Quadro 2 apresenta a lista dos artigos inclusos a partir da aplicabilidade dos critérios de inclusão e exclusão previamente descritos no item metodologia.

Quadro 2- Artigos utilizados para a construção desse estudo após a submissão aos critérios de inclusão e exclusão.

\begin{tabular}{|c|c|c|c|c|c|}
\hline N & TÍTULO & ANO & AUTORES & $\begin{array}{c}\text { PALAVRAS- } \\
\text { CHAVE }\end{array}$ & $\begin{array}{c}\text { TIPO DE } \\
\text { PESQUISA }\end{array}$ \\
\hline $\mathbf{1}$ & $\begin{array}{l}\text { O papel do psicólogo } \\
\text { hospitalar }\end{array}$ & 2014 & $\begin{array}{c}\text { FADINI, João } \\
\text { Paulo; } \\
\text { MEIADO, }\end{array}$ & $\begin{array}{l}\text { Psicologia } \\
\text { hospitalar. }\end{array}$ & $\begin{array}{c}\text { Estudo } \\
\text { bibliográfico }\end{array}$ \\
\hline
\end{tabular}




\begin{tabular}{|c|c|c|c|c|c|}
\hline & $\begin{array}{l}\text { atualidade: um } \\
\text { estudo investigativo }\end{array}$ & & $\begin{array}{l}\text { Adriana } \\
\text { Campos. }\end{array}$ & $\begin{array}{l}\text { Psicologia da } \\
\text { saúde. Psicólogo } \\
\text { hospitalar. }\end{array}$ & \\
\hline 2 & 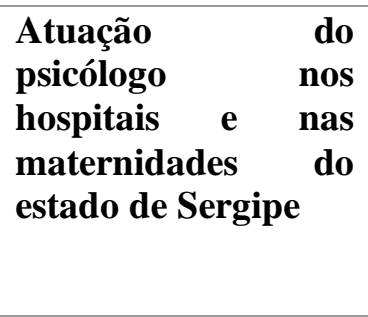 & 2012 & $\begin{array}{l}\text { SANTOS, Lyvia } \\
\text { de Jesus; } \\
\text { VIEIRA, Maria } \\
\text { Jésia. }\end{array}$ & $\begin{array}{l}\text { Atuação em } \\
\text { Psicologia, } \\
\text { Psicologia } \\
\text { hospitalar, Prática } \\
\text { profissional, } \\
\text { Trabalho em } \\
\text { saúde }\end{array}$ & Estudo de caso \\
\hline 3 & $\begin{array}{l}\text { Os desafios que os } \\
\text { psicólogos } \\
\text { hospitalares } \\
\text { encontram ao longo } \\
\text { de sua atuação }\end{array}$ & 2017 & $\begin{array}{l}\text { ALMEIDA, } \\
\text { Maria Lisbôa; } \\
\text { BRITO, Soraia } \\
\text { Silva; } \\
\text { MOSCON, } \\
\text { Daniela Campos } \\
\text { Bahia; SILVA, } \\
\text { Carla Souza } \\
\text { Ramos. }\end{array}$ & $\begin{array}{c}\text { Psicologia } \\
\text { Hospitalar; } \\
\text { Desafios; Atuação } \\
\text { do psicólogo }\end{array}$ & $\begin{array}{l}\text { Pesquisa } \\
\text { qualitativa }\end{array}$ \\
\hline 4 & $\begin{array}{lr}\text { A atuação } & \text { da } \\
\text { psicologia } & \\
\text { hospitalar, } & \text { breve } \\
\text { histórico e } & \text { seu } \\
\text { processo } & \text { de } \\
\text { formação no Brasil }\end{array}$ & 2019 & $\begin{array}{l}\text { ASSIS, Fabiane } \\
\text { Espindola; } \\
\text { FIGUEIREDO, } \\
\text { Sue Ellen } \\
\text { Ferreira } \\
\text { Modesto Rey. }\end{array}$ & $\begin{array}{c}\text { Psicologia; } \\
\text { Hospitalização; } \\
\text { Psicologia Médica; } \\
\text { Psicologia da } \\
\text { Saúde. }\end{array}$ & $\begin{array}{l}\text { Revisão } \\
\text { bibliográfica }\end{array}$ \\
\hline 5 & $\begin{array}{l}\text { O Psicólogo atuando } \\
\text { junto à criança } \\
\text { hospitalizada }\end{array}$ & 2013 & $\begin{array}{l}\text { JÚNIOR, José } \\
\text { Rodrigues } \\
\text { Rocha; } \\
\text { MESQUITA, } \\
\text { Darcilene de } \\
\text { Araujo; SILVA, } \\
\text { Edseany Pereira. }\end{array}$ & $\begin{array}{c}\text { Psicologia } \\
\text { Hospitalar. Atuacao } \\
\text { Psicologica. } \\
\text { Crianca } \\
\text { Hospitalizada }\end{array}$ & $\begin{array}{c}\text { Revisão } \\
\text { bibliográfica }\end{array}$ \\
\hline 6 & $\begin{array}{l}\text { Um estudo sobre o } \\
\text { papel do psicólogo } \\
\text { hospitalar }\end{array}$ & 2016 & $\begin{array}{l}\text { ABREU, } \\
\text { Angélica } \\
\text { Cristina } \\
\text { Oliveira; } \\
\text { ALVES, } \\
\text { Isabella } \\
\text { Drummond O. } \\
\text { Laterza; } \\
\text { COÊLHO, } \\
\text { Melissa } \\
\text { Batista; } \\
\text { FREITAS, } \\
\text { Amanda } \\
\text { Pereira } \\
\text { Barbosa; } \\
\text { PERES, Taís } \\
\text { Castro. }\end{array}$ & $\begin{array}{c}\text { Psicólogo no } \\
\text { hospital; } \\
\text { dificuldades } \\
\text { encontradas; } \\
\text { inserção na } \\
\text { instituição; saúde e } \\
\text { doença. }\end{array}$ & $\begin{array}{l}\text { Pesquisa } \\
\text { qualitativa }\end{array}$ \\
\hline
\end{tabular}

Fonte: Autoria própria. 
De acordo com Salman e Pulaskas (2013), a inserção do psicólogo no contexto hospitalar amplia de forma significativa o processo de humanização do atendimento ao paciente, que corresponde ao desenvolvimento de técnicas que enfatizem a valorização do indivíduo como um todo, o que leva o psicólogo a desenvolver a função de valorização do paciente, buscando fortalecer ele e seus familiares emocionalmente para o enfrentamento da doença, estando disposto a ouvir e dialogar sempre que possível.

Além disso, pode-se compreender também a partir da abordagem teórica levantada pelos autores que o apoio e acompanhamento do psicólogo hospitalar contribui de forma positiva diante da situação de sofrimento imposta pela enfermidade concorrendo para a promoção de um tratamento acolhedor e digno (SALMAN; PULASKAS, 2013).

Por outro lado, Chiattone (2011) enfatiza que o olhar do psicólogo aos pacientes hospitalizados é muito importante, valendo ressaltar alguns aspectos indispensáveis nesse processo de resolução de conflitos emocionais provenientes pelo enfrentamento da doença. Que são eles: atitudes do paciente frente a vida, os traços de personalidade, o grau de integração psíquica, as crenças que ele possui sobre a doença, sua maturidade interna, suas perdas significativas, etc.

Diante disso, pode-se evidenciar que o suporte psicológico ao paciente, e aos familiares é essencial, o que permite compreender a relevância do psicólogo hospitalar para a promoção do auxílio ao tratamento e a compreensão da internação e do adoecimento, assim como também em situações de óbito.

O espaço de atuação do psicólogo hospitalar expande-se amplamente a cada demanda. Compreendendo que a psicologia hospitalar lida com elementos que não podem ser controlados, até porque, seu trabalho envolve, além do paciente e familiares todos os profissionais que constituem a equipe multiprofissional, o que exige a sua integração com essa equipe interdisciplinar.

Conforme Carvalho (2013, p.361):

$$
\begin{aligned}
& \text { O desafio que se apresenta ao trabalho } \\
& \text { do Psicólogo no Hospital seria } \\
& \text { oferecer um modelo de formação para } \\
& \text { o psicólogo da saúde no contexto } \\
& \text { hospitalar fundamentado em um } \\
& \text { modelo de atenção integral à saúde. }
\end{aligned}
$$

Mesmo diante desses desafios é necessário que se possa buscar compreender o ser biopsicossocial no âmbito da saúde. Sendo extremamente relevante a participação de todos os integrantes da equipe para que $o$ atendimento hospitalar seja realizado de 
forma eficiente e humanizado (FOSSI; GUARESCHI, 2004).

É no hospital que o psicólogo intervém como mediador, na perspectiva de compreender a relação existente entre a equipe e a família e entre a equipe e o paciente. Pois, a equipe e a família executam papéis bastante relevantes no enfrentamento da doença e realização do tratamento.

Assim a presença do psicólogo no hospital torna-se fundamental para o paciente e seus familiares, pois ele mediara o contato entre o paciente e sua família, juntamente com a equipe de saúde, objetivando a facilitação da comunicação entre ambos, a fim de que contribuam durante $o$ tratamento do paciente (SALDANHA; BADCH; CRUZ, 2013).

Em suma, a intervenção do psicólogo em unidades hospitalares de urgência, se dá posteriormente a estabilizações das funções vitais da pessoa interna, dando assistência psicológica no intuito de auxiliar no tratamento dos fatores psicossociais e também na sua reabilitação, por meio do oferecimento de acolhimento do paciente, familiar/acompanhante e a equipe multidisciplinar.

Em seu estudo, Vieira identificou apenas um único profissional que atuava no serviço de urgência no maior hospital pública do estado de Sergipe. Dentre os diversos tipos de atendimentos que se enquadram o acolhimento é o mais atenuante.

Identificou-se ainda com esse estudo, que o atendimento psicológico ao paciente que se nega ao recebimento de algum tipo de cuidado prestado pela equipe multiprofissional, assim como também, ao acompanhamento específico por familiar em situação de óbito, etc.

\section{O papel do psicólogo no contexto hospitalar}

O papel do psicólogo hospitalar na relação com o paciente interno e seus familiares, responsáveis e acompanhantes, se concretiza a partir do estabelecimento do vínculo assistencial permitindo a identificação dos núcleos de sentido:

\footnotetext{
'receptividade', 'cooperação' e 'relação empática', esta última referida à questão de afetividade e percepção do paciente, familiar e/ou equipe para com o psicólogo. Esta situação permite ao profissional observar reações emocionais do outro, favorecendo uma maior interação na psicodinâmica do processo terapêutico 2,8,13.
}

Assim, percebe-se que o trabalho do psicólogo na área da saúde pública, em diversas localidades em todo o país tem se instituído como tema de diversos debates, conforme predispõe o Conselho Federal de Psicologia, tendo em vista que nos últimos 
anos a saúde mental tem sido bastante discutida.

Desse modo, pode-se observar que ainda há inúmeras instituições hospitalares

\section{CONCLUSÃO}

A partir da realização desse estudo, pode-se compreender o processo de atuação do psicólogo no contexto hospitalar, assim como também, a identificação histórica desse profissional, bem como os desdobramentos que se dão a partir daí, tendo em vista que o principal objetivo do psicólogo hospitalar é ajudar o paciente no seu processo de adoecimento, a fim de amenizar o sofrimento advindo da hospitalização. Além disso, objetiva também, perceber os sentimentos que são gerados e causados pelo internamento hospitalar, a fim de promover o bem-estar ao paciente e aos seus familiares.

Pode-se perceber ainda que os familiares cumprem um papel de extrema relevância no processo de tratamento contribuindo reciprocamente com o trabalho desenvolvido pelo psicólogo

\section{REFERÊNCIAS}

ALMEIDA, R. A.; MALAGRIS, L. E. N. (2015). Psicólogo da Saúde no Hospital Geral: um Estudo sobre a Atividade e a Formação do Psicólogo Hospitalar no Brasil. Psicol. 2015. que não dispõe de psicólogos para o atendimento da demanda de pacientes, o que tem inspirado diversos profissionais da saúde a pesquisar acerca desse tema.

hospitalar, sendo capaz de promover mobilizações para que o familiar interno possa posicionar-se de forma positiva diante do tratamento hospitalar recebido.

As condições que favorecem a integração do psicólogo hospitalar são inúmeras, bem como, são inúmeros os benefícios oferecidos pela presença desse profissional no âmbito hospitalar, por poder instigar em outros profissionais que integram a equipe interdisciplinar, a conscientização sobre a relevância de um trabalho em equipe, efetivamente integrado, no qual todos possam dedicassem ao máximo para que o tratamento possa apontar resultados positivos.

Pois essas atitudes são cruciais para que se possa permitir ao paciente hospitalizado o reconhecimento de seu valor, e de suas potencialidades, o que o estimulará progressivamente a rapidez na sua recuperação.

cienc. prof. [on-line]. 2015, 35 (3), 754-767, 2015. Disponível em:

<http://www.scielo.br/scielo.php?script=sc i_arttext\&pid=S1414-

98932015000300754\&lng=en\&nrm=iso>.

ANGERAMI-CAMON, V. A. (Org.). Psicologia da saúde: um novo significado 
para a prática clínica. 2. ed. São Paulo: Cengage Learning, 2011.

ALTAMIRA, Lorena L. A criança hospitalizada: um estudo sobre a atuacao do Psicologo Hospitalar. Monografia apresentada ao curso de Psicologia da Pontificia Universidade Catolica

de Minas Gerais. Arcos/MG 2010. Publicado em 07/01/2011. Disponivel em: <http://www.

webartigos.com/articles/56348/1/A-

crianca-hospitalizada-um-estudo-sobre-a-

atuacao-

-do-psicologo-hospitalar/pagina1.html>.

BAPTISTA, M. N. e DIAS, R. R. Psicologia Hospitalar: teoria, aplicacoes e casos clinicos. 2 ed.

Rio de Janeiro: Guanabara Koogan, 2010.

CASTRO, E. K. \& BORNHOLDT, E. Psicologia da Saúde $x$ Psicologia Hospitalar: Definições e Possibilidades de Inserção Profissional. Psicologia: Ciência e Profissão, Brasília, v. 24, n. 3, p. 48-57, 2004.

CHIATTONE, Heloisa Benevides de Carvalho. A Significação da Psicologia no Contexto Hospitalar. In: Psicologia da Saúde - um novo significado para a prática clínica. $2^{\mathrm{a}}$ Edição revista e ampliada. Cengage Learning Edições, p. $145-233,2011$.

CONSELHO FEDERAL DE PSICOLOGIA. Resolução do título profissional de especialista em psicologia. Brasilia, 2007.

CARVALHO, D.B. Psicologia da saúde crítica no contexto hospitalar. Psicol. cienc. prof. Brasília, v. 33, n. 2, 2013. Disponível em:

$<$ http://www.scielo.br/pdf/pcp/v33n2/v33n 2a08.pdf $>$.

MOTA, Roberta Araújo; MARTINS, Cileide Guedes de Melo; VERAS, Renata
Meira. Papel dos profissionais de saúde na política de humanização hospitalar. Psicol. estud., Maringá, v. 11, n. 2, Aug. 2006. Disponível em:

http://www.scielo.br/scielo.php?script=sci

_arttext\&pid=S1413-

$\overline{7} 3722006000200011 \& \operatorname{lng}=\mathrm{en} \& \mathrm{nrm}=\mathrm{isso}$

MOSIMANN, Laila T.; LUSTOSA, Maria Alice. A Psicologia hospitalar e o hospital. Santa

Casa da Misericórdia do RJ-CESANTA.

Rev. SBPH, Rio de Janeiro, v.14, n.1, jun. 2011.

ROMANO, B. Princípios para a Prática

Clínica em Hospitais. São Paulo: Casa do Psicólogo, 1999.

SEBASTIANI, R.W. Histórico e Evolução da Psicologia da Saúde Numa

Perspectiva Latino-americana. In V.A. Angerami-Camon (org.).

Psicologia da Saúde - Um Novo Significado Para a Prática Clínica. São

Paulo: Pioneira,2000.

SALMAN, Laila Abdul Karim; PAULAUSKAS, Davi Oscar Cabral.

Humanização em Unidade de Terapia Intensiva. Trabalho de Conclusão de

Curso da $5^{\text {a }}$ Turma de Pós-Graduação em Medicina Intensiva Adulta, do

Instituto Terzius e Faculdade Redentor, 2013.

SEBASTIANI, W.R., \& MAIA, C.M.E. Contribuições da psicologia da saúde hospitalar na atenção ao paciente cirúrgico. Recuperado em 4 de junho de 2010, da Scielo (Scientific Eletrocnic Library Online): http://www.scielo.br/scielo.php?script=sci _arttext\&pid=S0102-86502005000700010, 2005. > acesso em: 04 AGO. 2020.

TAVARES, Suyane Oliveira, et al. Interdisciplinaridade, Multidisciplinaridade Transdisciplinaridade. Curso de Psicologia do 
Centro Universitário Franciscano

(UNIFRA), Santa Maria, RS, Brasil, 2012.

Torezan Z. F. et al. (2013). A graduação em Psicologia prepara para o trabalho no hospital?. Psicologia: Ciência e Profissão, 33(1), 132-145. 